\title{
MedienPädagogik
}

Zeitschrift für Theorie und Praxis der Medienbildung

\section{Lernunterstützung mit digitalen Unterrichtsmaterialien}

\section{Interdisziplinäre Erkenntnisse und Entwicklungsperspektiven}

\author{
Richard Böhme und Meike Munser-Kiefer
}

\section{Zusammenfassung}

Digitalen Medien wird grosses Potenzial für die Lernunterstützung beigemessen, das bislang oft nicht ausgeschöpft wird. Dieser Beitrag gibt einen Einblick in den Forschungsstand zu differenziellen Effekten der Mediengestaltung als Orientierungs- und Entscheidungshilfe zur Auswahl bzw. zur Entwicklung lernförderlicher digitaler Unterrichtsmaterialien. Es werden spezifische Aspekte der Gestaltung von multimedialen, hypermedialen und ludischen Arrangements hinsichtlich des möglichen Einflusses auf die kognitiven Verarbeitungsprozesse beschrieben, Entwicklungsperspektiven aus dem Bereich der Intelligenten Tutoriellen Systeme aufgezeigt und jeweils empirische Befunde dazu berichtet. Dazu werden nach der Kognitiven Theorie des Multimedialen Lernens (1) inhaltsbedingte, (2) sachfremde und (3) lernrelevante kognitive Prozesse unterschieden. Es zeigt sich mit Blick auf die Lernvoraussetzungen, den Lerninhalt und das Lernziel ein komplexes Bedingungsgefüge, bei dem durch gezielte Modifikationen am digitalen Medium das Lernen optimiert werden kann. 


\title{
Learning support with digital teaching materials - Interdisciplinary findings and developmental perspectives
}

\begin{abstract}
Digital media is supposed to have great potential for learning support, which has not been fully exploited so far. This article provides an insight into the current state of research on the differential effects of media design: We describe specific aspects of design in multimedia, hypermedia and educational game-based arrangements and report possible influence on the cognitive processing and learning outcomes underpinned by results of empirical research. Additionally, we describe Intelligent Tutoring Systems as a means of optimizing learning processes in these different types of digital media design in the classroom. For this purpose, according to the Cognitive Theory of Multimedia Learning, we differentiate between (1) extraneous, (2) essential and (3) generative cognitive processes. With regard to the prerequisites for learning, the learning content and the learning objective, we show a complex set of conditions, in which learning can be optimized through targeted modifications to the digital medium.
\end{abstract}

\section{Theoretische Vorüberlegungen und leitende Grundannahmen}

Digitalen Medien wird u.a. lernunterstützende Funktion zugesprochen (KMK 2016, 5), da sie grosses Potenzial für die individuelle Unterstützung von Lernprozessen haben (Eickelmann und Vennemann 2013; Herzig 2014) - allerdings, wie hier gezeigt werden soll, unter der Voraussetzung, dass die Gestaltungsmerkmale und die Merkmale der Lernenden aufeinander abgestimmt werden. Der Begriff digitale Medien bezieht sich hier auf Kommunikationsmittel und Werkzeuge, die auf Basis digitaler Technik bzw. der algorithmischen Verarbeitung von Informationen funktionieren (in Anlehnung an Zorn 2011). Lernunterstützung unter Zuhilfenahme digitaler Technik wird dabei als Mikroadaption (vgl. z.B. Martschinke 2015) auf der Ebene der Lernprozesse verstanden, die darauf zielt, jede Schülerin und jeden Schüler mit ihren bzw. seinen individuellen Fähigkeiten optimal zu fördern. Oft unter Beibehaltung konventioneller Lernziele sollen diese so besser und für mehr Schülerinnen und Schüler erreichbar werden. 
Im Fokus stehen in diesem Beitrag fachübergreifende Gestaltungsmerkmale für einen breit aufgestellten Diskurs über eine lernförderliche «Verschaltung des Digitalen mit dem Analogen» (Baecker 2017, 3) in LehrLern-Arrangements. Beim Vergleich von häufig eingesetzten Lerntools (vgl. z.B. Huber et al. 2020) scheinen besonders drei Interaktionsformen im Vordergrund zu stehen, die hier die Struktur vorgeben: multimediale, hypermediale und ludische Arrangements. Zusätzlich werden neuere Entwicklungen zu Intelligenten Tutoriellen Systemen in den Blick genommen, da diese im Zusammenhang mit der individuellen Lernunterstützung einen emergenten Entwicklungs- und Forschungsbereich darstellen und in den drei Arrangements integriert werden können.

Obwohl in der Bildungswissenschaft digitalen Medien grosses Potenzial zur Lernunterstützung beigemessen wird (s.o.), fallen empirische Effekte eher gering aus (einen Überblick gibt Schaumburg 2015) und es wird oft beschrieben, dass digitale und konventionelle Unterrichtsmethoden zu ähnlichen Erfolgen führen (z.B. Hattie 2012). Dabei lassen sich diese in der unterrichtlichen Realität oft nicht trennen und Ursachen für potenzielle Unterschiede im Lernzuwachs finden sich nicht auf der Ebene der Sichtstruktur - analog und digital im Vergleich -, sondern auf der Ebene der Tiefenstruktur, den kognitiven Prozessen, die über das Medium als Mittler ausgelöst werden können (vgl. Oser und Baeriswyl 2001 sowie Renkl und Atkinson 2007). Ein Medium kann z.B. verschiedene Prozesse anregen: Eine rein textuelle und statische Inhaltsdarbietung in einem Print-Arbeitsbuch löst andere Lern- und Verarbeitungsprozesse aus, als wenn der gleiche Inhalt in einem digitalen Arbeitsbuch simuliert wird (vgl. Totter et al. 2020). Deshalb wird im Rückgriff auf die Kognitive Theorie des Multimedialen Lernens (Cognitive Theory of Multimedia Learning, CTML; Mayer 2009) herausgearbeitet, wie kognitive Prozesse mithilfe digitaler Technik auch unter Beibehaltung der Handlungspraxen und Lernziele mit Blick auf ihre lernunterstützendende Funktion optimiert werden können. In der CTML werden dazu drei Anforderungen an die kognitive Kapazität der Lernenden unterschieden: (1) Die sachfremde kognitive Verarbeitung (extraneous cognitive processing) bezieht sich auf Prozesse, die unabhängig vom Lerninhalt und aufgrund der Gestaltung des Mediums geleistet werden müssen. (2) Die inhaltsbedingte kognitive Verarbeitung (essential cognitive 
processing) umschreibt Prozesse, die in Aufbau und Struktur des Lerninhalts begründet liegen und diese die Lernenden etwa abhängig von ihrem Vorwissen unterschiedlich stark belastet. (3) Die lernrelevante kognitive Verarbeitung (generative cognitive processing) bezieht sich auf die Prozesse im Arbeitsgedächtnis, in dem Informationen aktiv gehalten werden müssen, um Wissen zu konstruieren und zu organisieren (Mayer 2014). In Anlehnung an die CTML gilt für komplex arrangierte Mediengestaltung, die sachfremde kognitive Verarbeitung zu reduzieren, die inhaltsbedingte kognitive Verarbeitung zu steuern und die lernrelevante kognitive Verarbeitung zu fördern.

Im Folgenden werden Gestaltungsmerkmale betrachtet, die sich von rein analogen Möglichkeiten unterscheiden und im Vergleich dazu in mindestens einem der Verarbeitungsprozesse abweichen. Beispiele illustrieren die kognitiven Anforderungen der CTML und werden durch empirische Befunde untermauert sowie zum Abschluss der Abschnitte stichpunktartig zusammengefasst. Dies soll Orientierungs- und Entscheidungshilfe geben, unter welchen Voraussetzungen ein entsprechendes Arrangement sinnvoll sein kann - wobei das nur grundlegende Prinzipien und Hinweise sein können, die nicht als präskriptive Handlungsempfehlungen zu interpretieren sind. Abschliessend wird ein Einblick in Intelligente Tutorielle Systeme gegeben und das Potenzial aufgezeigt, das sich daraus perspektivisch ergeben könnte.

\section{Digitale Lehr-Lern-Arrangements}

\subsection{Multimediales Arrangement}

Digitale Technik bietet die Möglichkeit, komplex arrangierte multimediale Lernmaterialien zusammenzustellen und zu entwickeln, die schriftliche (1) und/oder mündliche Texte (2), statische (3) und/oder bewegte Bilder (4) sowie akustische (5) und taktile Signale (6) integrieren. So ist es z.B. mit entsprechenden Lerntools möglich, Schülerinnen und Schüler einen Inhalt näherzubringen, indem dieser in einem persistent-schriftlichen Text wie einem niedergeschriebenen Sachtext (1) beschrieben ist. Der Inhalt wird 
an einem statischen Schema (3) strukturiert und in einer Simulation (4) veranschaulicht. Die Schülerinnen und Schüler bearbeiten dazu eine Aufgabe, die über einen transient-mündlichen Text wie eine eingesprochene Arbeitsanweisung oder strukturelle Hilfen (2) erläutert wird. Je nach Aufgabentyp und Algorithmus kann den Schülerinnen und Schülern dann ein direktes Feedback gegeben werden, das von einem einfachen Piepen (5) oder Vibrieren (6) für richtige und falsche Antworten bis hin zu elaborierten schriftlichen (1) oder mündlichen Hinweisen (2) zum Lösungsweg reicht.

Neben dieser eher auf Rezeption ausgerichteten Art der multimedialen Integration kann der Blick auch gezielt auf kreativ-produzierende Handlungen durch Schülerinnen und Schüler gerichtet sein.

\subsubsection{Transiente und persistente Bild- und Text-Formate}

Das multimediale Zusammenwirken kann sich bei Bildschirmmedien hinsichtlich der Darbietung von transienten Informationen wie gesprochenem oder kurzzeitig eingeblendetem Text sowie animierten Bildern von analogen Medien unterscheiden. Unter Annahme eines visuellen und eines akustischen Verarbeitungskanals wie sie in der CTML postuliert werden (Dual-Channel Assumption), kann bei Bildschirmmedien eine Entlastung in der Informationsverarbeitung angenommen werden, sofern der Text zu einem kohärenten Bild mündlich dargeboten wird. Wenn z.B. beim Thema «Wasserkreislauf» ein entsprechendes (komplexitätsreduziertes) Bild dargestellt ist, zu dem eine mündliche Erklärung gegeben wird, scheint dies effektiver zu sein, als wenn die Erklärung verschriftet ist. Dieser Effekt wurde mehrfach bei unterschiedlichen Altersgruppen nachgewiesen (z.B. Herrlinger et al. 2017; Metaanalyse von Ginns 2005). Allerdings müssen mündliche Texte zur weiteren Verarbeitung im Arbeitsgedächtnis aktiv gehalten werden, wodurch sich die lernrelevanten kognitiven Verarbeitungsprozesse erhöhen können (siehe dazu Diskussionsbeitrag von Schüler, Scheiter und Schmidt-Weigand 2011). Herrlinger (2012) konnte - anders als in der Studie von 2017 - keinen Vorteil einer Kombination aus mündlichem Text und Bild feststellen und mutmasst, dass dies an den unterschiedlich langen Sequenzen und den für die Lernenden unterschiedlich 
anspruchsvollen Inhalten lag. Anspruchsvolle Texte stellen unter der transient-textuellen Bedingung eine noch höhere Anforderung an die lernrelevanten kognitiven Ressourcen. Wenn eine Schülerin bzw. ein Schüler etwa beim Thema «Wasserkreislauf» noch wenig Vorwissen hat, dieses Thema nun aber unter dem Gesichtspunkt der globalen Erderwärmung verhandelt werden soll, und die Erklärung dadurch insgesamt komplex und lang wird, scheint diese in mündlicher Form weniger sinnvoll zu sein. Dies steht in Übereinstimmung zu Effekten sowohl in der Primarstufe (Leahy und Sweller 2011) als auch der Sekundarstufe (Wong et al. 2012). Um die Anforderungen an die lernrelevante und inhaltsbedingte kognitive Verarbeitung zu reduzieren, erweist es sich grundsätzlich und subgruppenübergreifend als hilfreich, Sachverhalte in inhaltlich bedeutsame Abschnitte zu segmentieren, sodass die Lernenden diese in ihrer individuellen Abruf- und Verarbeitungsgeschwindigkeit nutzen können (vgl. Hasler, Kersten und Sweller 2007). Zusätzlich können Hervorhebungen visueller, akustischer oder taktiler Art die Aufmerksamkeit der Lernenden lenken - das heisst, die inhaltsbedingte Verarbeitung wird gesteuert (für die Primar- und Sekundarstufe siehe z.B. Rouet et al. 2011). So wäre es beim Thema «Wasserkreislauf» denkbar, zunächst Wasserspeicher zu thematisieren, anschliessend auf deren Ursprung zu fokussieren und abschliessend den Kreislauf aufzuzeigen und dabei Hinweispfeile sequenziell einzubringen. Bei Schülerinnen und Schülern mit wenig Vorwissen empfehlen sich zudem Pausen, um metareflexive Prozesse gezielt anzuregen (Hasler, Kersten und Sweller 2007).

Empirische Belege deuten ferner darauf hin, dass im Sinne der DualChannel Assumption Animationen in kürzeren Einheiten die inhaltsbedingte kognitive Verarbeitung steuern können (z.B. Barak und Dori 2011) - etwa beim Aufzeigen des Kreislaufs im dritten Sinnabschnitt des Beispiels -, da die Kontextualisierung eines abstrakten Inhalts und Vorstrukturierung von Sequenzen es erleichtern, Schlüsse zu ziehen.

Das Vorwissen scheint sich mit Blick auf das Zusammenspiel aus Bild und Text auf die kognitiven Verarbeitungsprozesse auszuwirken: So legen die Befunde der Metaanalyse von Adesope und Nesbit (2012) nahe, dass Lernende mit geringem Vorwissen von einer redundanten visuell-auditiven Darbietung der Information profitieren ( $g=0.29 ; p<.05)$, wohingegen Schülerinnen und Schüler mit hohem Vorwissen keinen Vorteil davon 
haben ( $g=-0.05 ; p>$.05). Beim Beispiel «Wasserkreislauf» erscheint es demnach lediglich für Schülerinnen und Schüler mit wenig Vorwissen sinnvoll zu sein, Bildmaterial anzubieten, das genau die Informationen zum Wasserkreislauf enthält, die auch schon im Text vorkommen. Es ist anzunehmen, dass der visuelle Kanal zwar bei beiden Gruppen durch die parallele Verarbeitung von Bild und geschriebenem Text belastet wird, aber das Bild bei Schülerinnen und Schülern mit geringem Vorwissen kompensatorische Wirkung hat - das Bild zum Verständnis des Textes beiträgt - und die inhaltsbedingte kognitive Verarbeitung dadurch gesteuert wird. Bei Lernenden mit hohem Vorwissen scheint das Bild hingegen zu erhöhter Aktivität der sachfremden kognitiven Verarbeitung zu führen: Sie integrieren ebenfalls die visuelle und textuelle Information, was wegen des hohen Vorwissens jedoch verzichtbar wäre und daher die Aufmerksamkeit ungünstig verschoben wird (vgl. Mayer 2009).

Zusammenfassend weisen die Befunde darauf hin, dass es bei der $\mathrm{Zu}$ sammenstellung und Entwicklung von digitalen multimedialen Arrangements für das Lernen der Schülerinnen und Schüler hilfreich ist, u.a. folgende Aspekte zu berücksichtigen:

- Kohärente Bild-Text-Zusammenstellungen sind besonders für Schülerinnen und Schüler mit geringem Vorwissen hilfreich. Schülerinnen und Schüler mit hohem Vorwissen ziehen daraus offenbar weder einen nennenswerten Nutzen noch behindert es sie wesentlich.

- Je komplexer ein Sachverhalt und je geringer das Vorwissen der Schülerinnen und Schüler ist, desto grösser ist der Vorteil schriftlicher Erklärungen gegenüber mündlichen Erklärungen. Mündliche Erklärungen sollten dabei visuell unterstützt werden.

- Komplexe und abstrakte Themen werden möglichst in kürzeren Sinnabschnitten dargeboten und dazu Reflexionsaufgaben angeboten.

- Hervorhebungen und kürzere Animationen sind wertvoll, um die Aufmerksamkeit zu steuern und Kontextualisierungen herzustellen. 


\subsubsection{Modi des Schreibens und Lesens}

Neben den Unterschieden in der Verarbeitung von Informationen von transienten im Vergleich zu persistenten Darstellungsformen ergeben sich auch für das Schreiben und Lesen von inhaltlich und gestalterisch nicht modifizierten Texten am Bildschirm im Vergleich zum Schreiben und Lesen auf Papier teils unterschiedliche Verarbeitungsprozesse. Nach dem $\mathrm{Pa}$ radigma der Embodied Cognition beeinflussen sich die visuelle, akustische und taktile Wahrnehmung sowie die Motorik und Kognition wechselseitig (Calvo und Gomila 2008).

Beim Vergleich des Schreibens mit Stift, per Hardwaretastatur und per virtueller Tastatur zeigen sich jeweils Unterschiede u.a. hinsichtlich der visuellen Fokussierung, der Motorik sowie der Planungs- und Überarbeitungsprozesse: So wird z.B. beim Handschreiben der Blick auf die Schreibausführung gerichtet. Im Gegensatz dazu fokussieren kompetente Schreibende unter Einsatz einer Hardwaretastatur überwiegend das aktuell zu schreibende Wort auf dem Bildschirm. Bei Verwendung einer virtuellen Tastatur wechselt der Blick wiederum zwischen dem aktuell zu schreibenden Wort und der virtuellen Tastatur. Dies ist zurückzuführen auf die hier beschränkten Möglichkeiten, die räumliche Lage und Grenzen der Tasten zu erfassen sowie automatisiert zu tippen. Beim Handschreiben werden im Kontrast dazu in der Regel Buchstabengruppen (strokes) raum-zeitlich und graphomotorisch ausgeführt. Weiterhin sind beim Handschreiben zumeist grössere Einheiten in den Planungs- und Überarbeitungsprozessen betroffen als beim Verwenden der Hardwaretastatur oder der virtuellen Tastatur. Das bedeutet konkret, dass Schülerinnen und Schüler beim Handschreiben einerseits motorisch vergleichsweise stark involviert sind, was z.B. beim Aufbau von robusten Wortschemata zur Speicherung von orthografisch korrekten Schreibungen hilfreich sein kann (vgl. Modell zur wortspezifischen Einprägung nach Wedel-Wolff 2011 sowie Studie von Mangen et al. 2015). Andererseits müssen Schülerinnen und Schüler beim Handschreiben bereits vorab und ggf. länger strukturieren, wie sie einen Text, Absatz, Satz oder Teilsatz aufbauen, was sie darin vermitteln möchten und wie die einzelnen Glieder angeordnet werden sollen. Es ist davon auszugehen, dass dies mehr lernrelevante kognitive Ressourcen als beim Tastaturschreiben beansprucht. Gleiches gilt für das 
Überarbeiten eines Textes oder Satzes: Beim Handschreiben überarbeiten Schülerinnen und Schüler in der Regel grössere Einheiten, um ein kohärentes Bild nicht zu gefährden. Dies kann zu einer intensiveren Auseinandersetzung mit dem Material führen, mit der potenziell erhöhte Verarbeitungsprozesse auf zwei Ebenen assoziiert sein können: eine (unerwünschte) erhöhte sachfremde kognitive Verarbeitung, da sich die Schülerinnen und Schüler verstärkt auf die Schreibhandlung konzentrieren; oder eine (erwünschte) erhöhte inhaltsbedingte kognitive Verarbeitung, da die Schülerinnen und Schüler den Inhalt über das Schreiben (wiederholt) strukturieren.

Das Handschreiben auf dem Tablet unterscheidet sich zum Handschreiben auf Papier hinsichtlich der glatteren Oberfläche, die eine höhere graphomotorische Kontrolle erfordert (Gerth et al. 2016). Bereits in der zweiten Klasse sind Schülerinnen und Schüler in der Lage, die graphomotorische Exekution an die glattere Oberfläche anzupassen (ebd.), sodass unter Rückbezug auf die Embodied Cognition keine wesentlichen Unterscheide in der Verarbeitung beim Schreiben auf dem Tablet im Vergleich zum Schreiben auf Papier zu erwarten sind.

In Bezug auf das Lesen konnten Mangen und Kuiken (2014) sowie Mangen, Olivier und Velay (2019) zeigen, dass Probandinnen und Probanden, die einen Text am Tablet bzw. mit E-Reader lasen, grössere Unsicherheiten im Hinblick auf die Chronologie und Temporalität der Ereignisse hatten. Entsprechend der Embodied Cognition kann angenommen werden, dass Ereignisse mit einem gedruckten Buch besser rekonstruiert werden können, da diese auch sensomotorisch prozessiert werden, was bei digitalen Endgeräten fehlt. In der Studie von Mangen, Olivier und Velay (2019) scheint dies jedoch keine Auswirkungen auf das Leseverständnis gehabt zu haben. In der Metastudie von Delgado et al. (2018) konnte hingegen ein signifikanter Gesamteffekt (Hedges' $g=-0.21 ; d c=-0.21$ ) festgestellt werden, wobei speziell das Zeitkontingent für das Textverständnis (von Sachtexten) von grösserer Bedeutung zu sein scheint als bei Texten auf Papier. Erklärungsansätze basieren einerseits auf der Shallowing-Hypothese, nach der Personen bei digitalen Texten mehr zu Skimming und Scanning tendieren. Andererseits weisen metakognitive Ansätze darauf hin, dass Lernende ihr Verständnis am Bildschirm überschätzen und ihr Monitoring weniger zuverlässig einschätzen (zsf. Sidi, Ophir und Ackerman 2016). Interventionsstudien lassen 
mutmassen, dass es beim digitalen Lesen hierfür zusätzlicher Trigger wie Hervorhebungen und Verständnisfragen bedarf, die dem konditionierten scannenden Lesen bei entsprechendem Lernziel entgegenwirken (ebd.).

Mit Blick auf die Entscheidung für oder gegen eine bestimmte Modalität und ein bestimmtes Medium beim Lesen und Schreiben stehen Lernziele und Nebenfaktoren im Vordergrund, die gegeneinander abzuwägen sind. So stellt sich z.B. die Frage, ob und in welchem Ausmass der Nachteil des Tastaturschreibens im Hinblick auf die Abrufleistung (auf Wortebene) nicht durch andere Vorteile digitaler Technik wie Autokorrektur und erweiterte Überarbeitungs- und Kooperationsmöglichkeiten bezüglich eines bestimmten Lernziels aufgewogen werden können. Umfasst das Lernziel etwa ausschliesslich ein Verständnis für einen Inhalt zu entwickeln, dürften die Autokorrektur und die erweiterten Überarbeitungsmöglichkeiten zur Steuerung der inhaltsbedingten kognitiven Verarbeitungsprozesse und der Reduktion der sachfremden kognitiven Verarbeitungsprozesse beitragen.

Zusammenfassend weisen die Befunde darauf hin, dass es in Bezug auf das Lesen und Schreiben bei der Zusammenstellung und Entwicklung von digitalen multimedialen Lernarrangements für das Lernen der Schülerinnen und Schüler hilfreich ist, u.a. folgende Aspekte zu berücksichtigen:

- Motorik und Kognition beeinflussen sich nach der Embodied Cognition wechselseitig.

- Beim Schreiben mit der Tastatur sind die Einheiten der Planungs- und Überarbeitungsprozesse kleiner als beim Schreiben von Hand.

- Die Abrufleistung von Wörtern ist beim Schreiben mit Tastatur geringer als beim Schreiben von Hand.

- Die Unterschiede in der Verarbeitung zwischen dem Handschreiben auf dem Tablet und auf Papier dürften vernachlässigbar sein.

- Die zeitliche Einordnung von Geschehnissen gelingt beim Lesen auf Papier besser als beim Lesen an einem Bildschirmmedium.

- Das Leseverständnis beim Lesen von Sachtexten auf Papier ist grösser als beim Lesen an einem Bildschirmmedium. Zur Kompensation sind zusätzliche Hervorhebungen und Möglichkeiten zur Selbstkontrolle von Bedeutung. 
Während ein chronologischer Verarbeitungsmodus bei bestimmten (digital) präsentierten Bild-Text-Kombinationen geboten ist (z.B. beim Lesen eines Sachtextes), weichen hypermediale Arrangements davon ab.

\subsection{Hypermediales Arrangement}

Informationen werden in Hypermedia überwiegend nichtsequenziell und in dynamischen Strukturen repräsentiert. Eine Subkategorie von Hypermedia sind Hypertexte, anhand derer im Folgenden Merkmale von Hypermedia beschrieben werden. Sie können unterschiedlich komplex gestaltet sein und von einfachen linearen über hierarchische bis hin zu vernetzten Hypertextstrukturen reichen (Unterscheidung nach Engebretsen 2000; siehe Abb. 1).

(1)

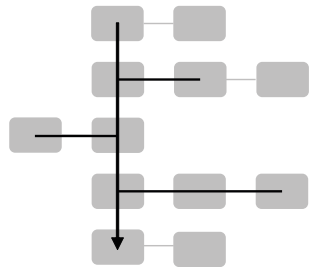

(2)

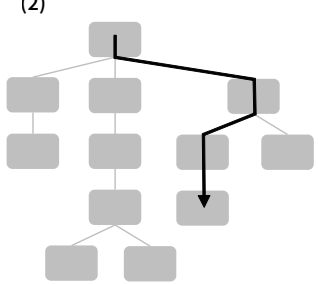

(3)

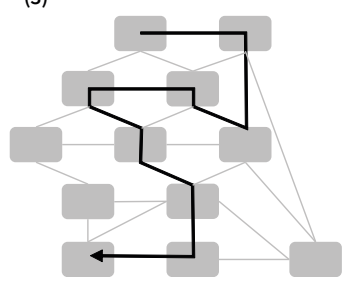

Abb. 1.: Schematische Darstellung von linearen (1), hierarchischen (2) und vernetzten (3) Hypertextstrukturen (Abbildung in Anlehnung an Engebretsen 2000).

Lineare Hypertexte ähneln stark der Struktur von gedruckten Texten, da hier die Präsentation von Informationen linear erfolgt (vgl. Abb. 1, [1]). Zu einzelnen Themenschwerpunkten können Zusatzinformationen dargeboten werden. Solche diskontinuierlichen Knoten weisen in linearen Hypertextstrukturen eine gleichartige Funktion wie z.B. gedruckte Lexika auf, die in der Regel nur bei unklaren Begriffen herangezogen werden. Im Unterschied dazu wird ein Verlassen des vorgesehenen Lernpfades bei linearen Hypertexten vermieden, da die Schülerinnen und Schüler auf den originären Pfad zurückkehren müssen, um in der Aufgabe fortzuschreiten. Beim Beispiel «Wasserkreislauf» wäre es nach einer solchen Struktur denkbar, die im Kapitel 2 skizzierte Gliederung Wasserspeicher > Ursprung der Wasserspeicher > Kreislauf > klimabedingte hydrologische Veränderungen 
linear darzubieten und zu den einzelnen Punkten zusätzlich (für das Thema relevante) Begriffsklärungen in Form von schriftlichen oder mündlichen Texten und/oder statischen oder bewegten Bildern anzubieten. Unter der Bedingung einer intuitiv-einfachen Gestaltung des User Interface können dadurch die sachfremden kognitiven Verarbeitungsprozesse vergleichsweise geringgehalten werden (vgl. Salmerón et al. 2018). Daher eignet sich ein solches Arrangement insbesondere für Schülerinnen und Schüler, die einen klar vorgegebenen Lernpfad und eine eng geführte Struktur benötigen, um deren inhaltsbedingte kognitive Verarbeitung zu steuern.

Anspruchsvoller sind hierarchische und vernetzte Hypertexte aufgebaut. Während hierarchische Hypertexte eine Baumstruktur aufweisen (vgl. Abb. $1,[2])$, wird die Information in vernetzen Hypertexten in dezentralen Netzwerken organisiert (vgl. Abb. 1, [3]). Sie haben im Unterschied zu linearen Hypertexten (und zu gedruckten Texten) keinen vorgegebenen kontinuierlichen Lernpfad, sondern ermöglichen es, zu vereinzelten Inhaltsbereichen zunehmend detaillierte Informationen zu bekommen. Beide Strukturen bergen für Schülerinnen und Schüler die Gefahr, sich von der ursprünglichen Aufgabenstellung und dem vorgesehen Lernpfad zu entfernen. Insbesondere bei der Arbeit mit vernetzen Hypertextstrukturen müssen die zu bearbeitenden Informationseinheiten im Sinne der Navigation (vgl. Voss 2006; Lawless und Schrader 2008) eigenständig ausgewählt und deren Reihenfolge festlegt werden, ohne vorab gesicherte Informationen über deren Zweckmässigkeit in Bezug auf die Aufgabe zu haben. Daher gleicht die Navigation in diesem Kontext dem Problemlösen (Brand-Gruwel, Wopereis und Walraven 2009), was für Schülerinnen und Schüler hohe Anforderungen an die inhaltsbedingte kognitive Verarbeitung und Selbstregulation stellt. Die Gliederung des Themas «Wasserkreislauf» könnte auch hier beibehalten werden, wobei die Gliederungspunkte von einem Ausgangstext in einer nicht vorab definierten Reihenfolge abgerufen werden können. Eine Schülerin bzw. ein Schüler könnte zunächst einen Text zum Ursprung von Wasserspeichern aufrufen, sich danach über Quellen und das Grundwasser kundig machen, dabei auf Informationen zu sinkenden Grundwasserspiegeln in verschiedenen Regionen zurückgreifen und so weiter. 
Die Verwendung solch vernetzter Strukturen scheint speziell dann förderlich zu sein, wenn ein flexibles, multiperspektivisches Verständnis eines komplexeren Sachverhalts aufgebaut werden soll (z.B. Spiro et al. 1992). Allerdings interagiert das Problemlösen als übergeordneter kognitiver Prozess mit diversen anderen kognitiven Prozessen, sodass das Navigieren einer hohen Arbeitsgedächtniskapazität bedarf. In Übereinstimmung dazu war ein entsprechendes Arrangement für Schülerinnen und Schüler (einer vierten Jahrgangsstufe) mit geringer Arbeitsgedächtniskapazität in einer Studie von Kornmann et al. (2016) nicht förderlicher für den Aufbau eines multiperspektivischen Denkens als in einer linearen Struktur. Kornmann et al. vermuten, dass bei Lernenden mit geringerer Arbeitsgedächtniskapazität übermässig viel kognitive Kapazität durch eine erhöhte sachfremde Verarbeitung blockiert ist und daher nicht genügend lernrelevante Verarbeitung prozessiert werden kann.

Wie sich bereits beim Lesen von einfachen (linearen) Texten auf einem Bildschirmmedium gezeigt hat, ist auch beim Lesen von Hypertexten der Aufbau von entsprechenden Strategien zur Reduktion von sachfremder kognitiver Verarbeitung und effektiver Navigation notwendig (Naumann und Salmerón 2016). Aussichtsreich erscheint die Kohärenzstrategie (siehe Salmerón, Kintsch und Cañas 2006; Salmerón et al. 2018): Nach dieser werden über semantische Kohärenz relevante Informationen selektiert und zyklisch verarbeitet, was das Textverständnis verbessert und das effektive Time-on-Task fördert (ebd.). Sie kann bereits in der Grundschule angebahnt werden (Salmerón und García 2011), indem die Aufmerksamkeit mithilfe von visuellen und/oder akustischen Signalisierungen auf die relevanten Aspekte gelenkt wird (siehe Rouet et al. 2011).

In vernetzten Hypertextstrukturen zum Thema «Wasserkreislauf» ohne vorgegebene Abrufreihenfolge könnte es zum Aufbau einer Kohärenzstrategie z.B. hilfreich sein, auf semantische Ähnlichkeit zwischen Aufgabenstellung und den relevanten Informationen im Text zu achten. Diese könnten zudem nach kurzer Bedenkzeit visuell hervorgehoben werden, um die Aufmerksamkeit zu steuern. Bei Klicks auf semantisch nahe als auch weit entfernte Verlinkungen könnten Hinweise oder Fragen zur Reflexion mit Bezug zur Aufgabenstellung einfügt werden, die vor dem Abrufen des verlinkten Textes beantwortet werden müssen - wie z.B. «Die 
Aufgabe bezieht sich auf den Wasserkreislauf. Wähle Fragen aus, die du mit diesem Text beantworten kannst! Antwort A, B, C, D.»

Als weniger erfolgreich in Bezug auf eine effektive Navigation erweisen sich die first-mentioned-Strategie, nach der die Verlinkungen in chronologischer Reihenfolge aufgerufen werden, und die interessensbezogeneexplorative Strategie (Salmerón, Kintsch und Cañas 2006; Salmerón et al. 2018), bei der die Verlinkungen unsystematisch nach Interesse abgerufen werden und eher von motivationaler Bedeutung sind.

Zusammenfassend weisen die Befunde darauf hin, dass es bei der $\mathrm{Zu}-$ sammenstellung und Entwicklung von digitalen hypermedialen Arrangements für das Lernen der Schülerinnen und Schüler hilfreich ist, u.a. folgende Aspekte zu berücksichtigen:

- Es kann für Schülerinnen und Schüler mit wenig Vorwissen zu anspruchsvoll sein, mit einer vernetzten Hypertextstruktur zu arbeiten.

- Leistungsstärkere Schülerinnen und Schüler können mithilfe vernetzter Strukturen im Bereich des Problemlösens gefördert werden.

- Für eine effektive Navigation können Kohärenzstrategien aufgebaut werden. Die semantische Ähnlichkeit zwischen Aufgabenstellung und relevantem Inhalt spielt dabei eine zentrale Rolle.

Ähnlich wie beim Hypertext-Lesen erfordern auch die unterschiedlichen Formen von Lernspielen verschiedene Lernvoraussetzungen. Diese werden im Folgenden näher betrachtet.

\subsection{Ludisches Arrangement}

Beim Konzept des (digitalen) spielbasierten Lernens (auch: [digital] Gamebased Learning) werden teils unter Zuhilfenahme von Intelligenten Tutoriellen Systemen (siehe Kapitel 3) lernrelevante Aufgaben in eine ludische (digitale) Umgebung eingebettet. Dabei wird grundsätzlich zwischen Gamification und Serious Games unterschieden (vgl. Deterding et al. 2011). Der Gamification sind eher einfach gehaltene ludische Gestaltungen und die Integration von Spielelementen zuzuordnen. Darunter fallen z.B. Belohnungselemente wie Auszeichnungen und Abzeichen sowie die Verwendung von Identifikationsfiguren. Gamification hat bereits vor einiger 
Zeit Einzug in die Klassenzimmer erhalten und findet sich in zahlreichen fachspezifischen und -unspezifischen Lerntools aller Jahrgangsstufen wieder. Gemeint sind hiermit z.B. Rechenaufgaben, bei denen «Rechenpokale» gewonnen werden können oder Geografie-Quiz-Aufgaben, bei denen eine Schülerin bzw. ein Schüler oder ein Team als Gewinnerin bzw. Gewinner hervorgeht. Serious Games stellen hingegen Spiele dar, die bis hin zu komplex aufbereiteten interaktiven und kooperativen Lernwelten reichen können. Damit sind z.B. Abenteuerspiele wie digitale Expeditionsreisen gemeint, auf denen alleine oder in einem Team, das ggf. mit anderen Teams konkurriert, (z.B. MINT-) Rätsel gelöst werden müssen (für ein Beispiel siehe Eckardt und Robra-Bissantz 2016). Auch für den Deutschunterricht gibt es Ansätze, bei denen etwa zur Förderung des Textverständnisses erweiterte Realitäten (Augmented Reality, AR) erschaffen werden. Bei TobarMuñoz, Baldiris und Fabregat (2017) «erwacht» z.B. die Szenerie des Textes, wenn das Tablet darauf gerichtet wird. In und zu dieser Szenerie werden dann Aufgaben gestellt, die sich auf den zuvor gelesenen Inhalt beziehen.

Für das Game-based Learning im Allgemeinen konnten in mehreren Metaanalysen positive Effekte in Bezug auf den Lernzuwachs unabhängig von der Altersgruppe und vom Geschlecht nachgewiesen werden (siehe z.B. Clark, Tanner-Smith und Killingsworth 2016; Vogel et al. 2006 sowie Wouters et al. 2013). Die differenziellen Effekte in Primärstudien sind jedoch weniger eindeutig (für einen Überblick siehe Wouters et al. 2013, Tab. 1). Dies gilt auch für den Bereich der Motivation (siehe ebd., Tab. 2), die sich auf das Engagement, die Art und Weise in der Auseinandersetzung mit dem Lernmaterial und folglich auf die Lernergebnisse auswirkt (siehe das erweiterte Erwartungs-Wert-Modell nach Wigfield und Eccles 1992) und deswegen als einer der zentralen Vorteile von Game-based Learning gesehen wird.

In Bezug auf den Lernzuwachs liegt ein Grund für die unterschiedlichen Ergebnisse möglicherweise in der Anlage der Primärstudien: Diese fokussieren oftmals auf Lernergebnisse niedriger Ordnung, sodass Lernziele höherer Ordnung nicht adäquat abgebildet werden. Zwar können sich Serious Games positiv auf die Behaltensleistung von Faktenwissen auswirken, da sie durch Animationen und die direkte Einbettung der Aufgabe in den Kontext zur Komplexitätsreduktion beitragen und dadurch 
die inhaltsbedingte kognitive Verarbeitung der Schülerinnen und Schüler gesteuert wird (vgl. Kapitel 2.1). Das Potenzial wird aber besonders darin gesehen, sie als Werkzeuge zur Simulation komplexer Verhältnisse zu nutzen (z.B. Squire, Gee und Jenkins 2011) und sie zum Erwerb entsprechender Problemlösestrategien und zum Aufbau sozial-kommunikativer Kompetenzen beim kollaborativen Arbeiten heranzuziehen. In Übereinstimmung dazu zeigen die Befunde von Wouters et al. (2013), dass situierte Lernspiele höhere positive Effekte auf das Problemlösen hatten als auf Drill-and-Practice-Anwendungen. In der Gesamtheit erweist sich dieser Erklärungsansatz aber als nicht ausreichend. Clark, Tanner-Smith und Killingsworth (2016) schlagen daher vor, den Blick zusätzlich auf konkrete Gestaltungsmerkmale zu richten und deren Wirkweisen zu berücksichtigen (ebenso Kapitel 2.1). Speziell bei Serious Games zeigte sich, dass narrative und bildlich komplexe bzw. visuell realistische Gestaltungen insgesamt weniger effektiv als schematisch aufgebaute Lernspiele sind (ebd.). Es kann angenommen werden, dass die «messiness der realen Welt» (Tobar-Muñoz, Baldiris und Fabregat 2017), die komplexe Arrangements imitieren, bei einem Grossteil der Schülerinnen und Schüler zu hohe Anforderungen an die lernrelevante kognitive Verarbeitung stellt, was gleichzeitig zu erhöhter sachfremder kognitiver Belastung führt. Es zeigt sich also ein ähnliches Bild wie bei der Verwendung von vernetzten Hypertextstrukturen (siehe dazu Kapitel 2.2).

Beim Thema «Wasserkreislauf» wäre es z.B. denkbar, eine forschende Figur den Weg eines Wassertropfens nachverfolgen zu lassen. Dabei lösen die Schülerinnen und Schüler Rätsel - etwa zur Beschaffenheit der Bodenschichten, durch die das Wasser sickert bzw. auf der sich das Grundwasser sammelt. Die animierten Bilder können dabei schematisiert und reduziert werden, indem sich die Figur von einem Ausgangsbild in verschiedene Ausschnitte dieses Bildes begibt, in denen die relevanten Informationen hervorgehoben sind - wie z.B. klar voneinander abgegrenzte Erdschichten oder sichtbarer Wasserdampf. Die Spielmodi können dabei unterschiedlich gestaltet sein. Clark, Tanner-Smith und Killingsworth (2016) konnten zeigen, dass Spielmodi, bei denen einzelne Schülerinnen und Schüler miteinander konkurrieren, nicht lernförderlicher als herkömmliche Unterrichtsmethoden sind. Als Grund wird gesehen, dass das 
erhöhte Kompetenzerleben einer Schülerin bzw. eines Schülers mit einem verminderten Kompetenzerleben der bzw. des jeweils anderen einhergeht und sich die spielbasierten Vorteile dadurch nivellieren. Die verminderte Motivation wirkte sich indirekt auf die Lernergebnisse aus.

Das Kompetenzerleben ist neben dem Autonomieerleben und der sozialen Eingebundenheit Teil der Basic Human Needs nach der Selbstbestimmungstheorie von Deci und Ryan (1985). Werden sie befriedigt, kann dies den Aufbau von Interesse und Motivation von Schülerinnen und Schülern unterstützen und das Lernen fördern. Durch den Einsatz bestimmter Gestaltungsmerkmale beim Game-based Learning kann es gelingen die $\mathrm{Ba}$ sic Human Needs zu befriedigen (vgl. Birk et al. 2016): So kann das Autonomieerleben nicht nur dadurch gefördert werden, dass Schülerinnen und Schüler ein realistisches Problem eigenständig und auf einem eigenen, ggf. vorstrukturierten Lernpfad (vgl. Kapitel 2.2) bearbeiten können, sondern z.B. auch durch die Möglichkeit, sich eine Identifikationsfigur zusammenzustellen (siehe Birk et al. 2016). Das Erleben von sozialer Eingebundenheit kann durch das Zusammenarbeiten in Teams hervorgerufen werden, die das Problem miteinander lösen und die aufeinander angewiesen sind, weil sie unterschiedliche Rollen, Kompetenzen und Aufgaben haben. Kompetenzerleben kann wiederum dadurch gestärkt werden, dass solche Rollen- und Kompetenzverteilungen auf die individuellen Fähigkeiten und Fertigkeiten der Lernenden angepasst sind und alle Lernenden Auszeichnungen und Belohnungen für das Geleistete erhalten können (vgl. Spires et al. 2011). Dabei scheinen sowohl einfache Auszeichnungen (einfache Punktvergabe oder Kennzeichnung der Lernaktivitäten) als auch komplexere Belohnungssysteme (wie das Freischalten von besonderen Identifikationsfiguren o.ä.) der Lernleistung über die Motivation zuträglich zu sein (ebd.). Bemerkenswert ist dabei, dass sich bei Serious Games, die extrinsische Anreize (wie etwa durch Auszeichnungen) schaffen, keine negative Entwicklung der intrinsischen Motivation abzuzeichnen scheint (Wouters et al. 2013; Clark, TannerSmith und Killingsworth 2016; vgl. auch Ryan, Rigby und Przybylski 2006). Womöglich ergibt sich dieser Effekt durch die gleichzeitige, vergleichsweise hohe Befriedigung der anderen Basic Human Needs.

Demnach könnte es z.B. sinnvoll sein, die Rätsel zum Wasserkreislauf in einem Forschenden-Team zu lösen: Um zu überprüfen, ob ein 
Wassertropfen bei unterschiedlichen Temperaturen immer denselben Weg geht, könnten die einzelnen Schülerinnen und Schüler dieses Teams unter den entsprechenden Bedingungen testen und die Ergebnisse im «Expertinnen- und Expertengremium» diskutieren. Bei richtigen Antworten könnte für das Team als Belohnung eine Auswahl an besserem oder zusätzlichem Equipment wie einem Motorboot statt einem Paddelboot oder neue Kleidung freigeschaltet werden.

Zusammenfassend weisen die Befunde darauf hin, dass es bei der $\mathrm{Zu}-$ sammenstellung und Entwicklung von digitalen ludischen Arrangements für das Lernen der Schülerinnen und Schüler hilfreich ist, u.a. folgende Aspekte zu berücksichtigen:

- Speziell bei Serious Games ist die multimediale Zusammensetzung für die kognitive Verarbeitung von Bedeutung: Während Schülerinnen und Schüler mit geringem Vorwissen von komplexitätsreduzierenden Arrangements profitieren können, können komplexe Arrangements bei Schülerinnen und Schüler mit hohem Vorwissen multiperspektivisches Denken anregen.

- Besondere Bedeutung wird dem Game-based Learning in Bezug auf die Motivation beigemessen. Gestaltungsmerkmale und Sozialformen auf die Befriedigung der Basic Human Needs auszurichten, kann ein ludisches Arrangement motivierend aufbereiten.

- Im Allgemeinen kann empfohlen werden, sowohl die ludische Aufbereitung als auch das Belohnungssystem nicht zu aufwändig zu gestalten, weil die Effekte entweder ähnlich hoch ausfallen oder zu komplexe Gestaltungen die sachfremde kognitive Verarbeitung erhöhen.

Auch wenn die Passung zwischen den Anforderungen in Serious Games und den Fähigkeiten der Lernenden ein wesentliches Kriterium für deren Lernförderlichkeit ist, gelingt dies häufig noch nicht in einem zufriedenstellenden Masse. Um diese Form der individuellen Lernunterstützung noch besser zu erreichen, werden aktuell einige Intelligente Tutorielle Systeme entwickelt und erprobt. 


\section{Intelligente Tutorielle Systeme}

Individuelle Lernunterstützung kann im Sinne des formativen Assessment besonders gut erreicht werden, wenn eine Verknüpfung und Passung von (1) (standardisierter) Lernverlaufsdiagnostik, (2) adaptiv-aufgabenbezogenem Feedback und (3) adaptiver Förderung für die einzelnen Lernenden gelingt (vgl. z.B. Hattie 2012; Black und Wiliam 2009; Schmidt und Liebers 2015; zum Feedback siehe auch Hattie und Timperley 2007). Die Implementation und das kontinuierliche Anwenden dieser formativen Sequenzen ist insbesondere bei heterogener Schülerschaft herausfordernd (siehe Souvignier und Hasselhorn 2018), sodass formatives Assessment (ohne digitale Unterstützung) eher im Expertensetting bzw. mit Expertencoaching und nicht im ökologisch validen Setting zu gelingen scheint (vgl. ebd.; siehe auch Wang, Gennari und Waxman 1985; Franke-Braun 2008). Intelligente Tutorielle Systeme (ITS) haben das Potenzial, hier zu unterstützen. Sie werden bereits seit den 1960er-Jahren entwickelt (für einen Überblick siehe Nkambou et al. 2010), um mithilfe digitaler Technik «die Auswertung einzelner Schritte innerhalb des Lösungsprozesses, Rückmeldung zu einzelnen Lösungsschritten, kontextspezifische Hinweise für den nächsten Schritt [...] sowie eine individualisierte Aufgabenauswahl [automatisiert zu ermöglichen].» (Schütze, Souvignier und Hasselhorn 2018, 705f).

Trotz des theoretischen Potenzials konnte in Metaanalysen bis dato nur eine ähnlich hohe lern- bzw. leistungsförderliche Wirkung von ITS festgestellt werden wie beim ausschliesslichen Tutoring durch Lehrkräfte (siehe z.B. Kulik und Fletcher 2016; Ma et al. 2014; Steenberg-Hu und Cooper 2014). Allerdings beruhen die in ITS verwendeten Algorithmen häufig auf frequentistischen Ansätzen nach dem Allgemeinen oder Verallgemeinerten Linearen Modell, u.a. aufgrund eingeschränkter computationaler Ressourcen für statistische Analysen. Da diesen Ansätzen strenge Modellannahmen zugrunde liegen, im Bildungskontext aber eine Vielzahl an Variablen berücksichtigt werden müssen, konnte Formatives Assessment mit ITS - ebenso wie durch Lehrkräfte - noch nicht optimal umgesetzt werden (für einen Überblick siehe Efron und Hastie 2016). Mit dem Anstieg der Rechenleistung sind die Möglichkeiten inzwischen vielfältiger geworden, sodass Ansätze aus dem Bereich Machine Learning (ML) - die bei der Analyse grosser Datensätze die geeignetere Alternative zu 
klassisch-frequentistischen Modellen sind - verstärkt eingesetzt werden. Auch steht die fehlende Interpretierbarkeit von Algorithmen nun im Fokus der Forschungs- und Entwicklungsarbeit (siehe z.B. Molnar 2018), die die Verbreitung von ML-basierten ITS längere Zeit hemmten (vgl. Doshi-Velez und Kim 2017).

Aus diesen Gründen ist die empirische Befundlage zur lernunterstützenden Wirkung von ML-basierten ITS bisher marginal. Erste Befunde zeigen, dass mit ML-basierten ITS auch offene Antwortformate qualitativ so auszuwerten sind, dass die Interpretation inhaltlich korrekt und ähnlich genau wie die von menschlichen Expertinnen und Experten sind (siehe z.B. Vögelin et al. 2018; Zehner, Sälzer und Goldhammer 2016). Dazu werden offene Antworten auf bestimmte Fragen etwa über Latente Semantische Analysen (LSA) computational verarbeitet, die dann Antwort-Clustern zugeordnet werden können. Dies ist die Voraussetzung dafür, dass adaptives maschinelles Feedback gegeben werden kann. Befunde weisen darauf hin, dass solche Feedbacks mit ML-basierten ITS umgesetzt werden können und sie sich positiv auf die Lernentwicklung von Schülerinnen und Schülern auswirken (z.B. Meurers et al. 2019).

Empirische Befunde - insbesondere zur automatisierten Anpassung der Aufgabenschwierigkeit, des Formats und des Inhalts - stehen noch aus.

Beim Thema «Wasserkreislauf» wäre es (sowohl im Beispiel aus dem hypermedialen oder aus dem ludischen Arrangement) möglich, einen MLAlgorithmus zu trainieren, der auf Antworten der Schülerinnen und Schüler zu den Aufgaben bzw. Rätseln ein Feedback gibt. Vereinfacht dargestellt: Wenn z.B. auf die Frage «Warum versickert das Wasser nicht immer tiefer im Boden?» die Antwort gegeben wird «Weil die Schichten im Boden ganz weit unten gefroren sind.», wird diese dann etwa über LSA verarbeitet und anschliessend dem Cluster «Fehlvorstellung + irrelevantes Konzept + Hinweis auf Beschaffenheit notwendig» zugeordnet. Zu diesem haben Expertinnen und Experten aus dem Fachbereich wiederum die Rückmeldung vorbereitet «Gute Idee, aber hier stimmt sie nicht. Schaue dir hier einmal das Experiment an [Verlinkung].», die den Lernenden eine passgenaue Weiterarbeit ermöglicht. 
Insgesamt ist hervorzuheben, dass ITS weder in Konkurrenz zur Aufgabe der Lehrkraft stehen noch Unterricht oder die kognitiven Prozesse aller Lernenden grundsätzlich verändern. Vielmehr können sie als Werkzeuge für Lehrkräfte fungieren und sie dabei unterstützen, Lerninhalte in unterschiedlicher Komplexität anzubieten, das Format und die Rückmeldung auf die Bedürfnisse der Lernenden anzupassen und Schülerinnen und Schülern unmittelbar nach der Bearbeitung von Aufgaben eine Rückmeldung dazu zu geben. Dadurch dürfte die Steuerung der inhaltsbedingten kognitiven Verarbeitung insbesondere der Schülerinnen und Schüler positiv beeinflusst werden, die unter- oder überfordert sind und/oder z.B. aus Kapazitätsgründen nur selten unmittelbares Feedback bekommen.

\section{4. $\quad$ Fazit und Perspektiven}

Digitale Medien haben das Potenzial, individuelle Lernprozesse zu optimieren und konventionelle Lernziele für mehr Schülerinnen und Schüler erreichbar zu machen. Doch nicht das Medium oder die Technik an sich bewirken eine Verbesserung; es geht vielmehr um die effektive Nutzung der erweiterten Möglichkeiten, günstige kognitive Verarbeitungsprozesse anzuregen. Zentral dafür ist die Orchestrierung des Medienarrangements mit Blick auf Lernziele und Lernbedürfnisse der einzelnen Lernenden. Befunde zu spezifischen kognitiven Verarbeitungsprozessen lassen erwarten, dass ein Mehr an digital umgesetzten gestalterischen Elementen und Funktionen auf der Ebene der Sichtstruktur nicht unbedingt zu besserem Lernen führt. Vielmehr sollten spezifische Effekte auf der Ebene der Tiefenstruktur bei der Entwicklung, dem Einsatz und bei der Erforschung digitaler Unterrichtsmaterialien berücksichtigt werden. Dies erfordert eine Synthese der Erkenntnisse der klassischen Lehr-Lern-Forschung (z.B. konstruktivistischer Lernbegriff, instruktionspsychologische Grundlagen, Unterrichtsqualitätskriterien, Erkenntnisse zu Motivation und Einstellungen) einerseits und der Erkenntnisse zum multimedialen Lernen (z.B. strukturelle medienpsychologische Analysen zu differenziellen Effekten der Mediengestaltung und des -einsatzes) andererseits. 
Perspektivisch erscheint es sinnvoll, zusätzlich die umfassenderen Möglichkeiten des Machine Learnings zu nutzen und diese in die bestehenden Formen digitaler Unterrichtsmaterialien zu integrieren und dadurch die Lernangebote durch Machine Learning-basierte Intelligente Tutorielle Systeme zu optimieren. Weiterhin sind die Möglichkeiten der Förderung hierarchiehöherer Prozesse wie z.B. Problemlösen und Transfer in kollaborativen Serious Games- und Game-based Learning-Settings noch nicht ausgeschöpft. Das betrifft in gleicher Weise das kontextualisierte Lernen, das durch Immersion und Situierung aus theoretischer Perspektive grosses Potenzial für die Verbesserung individueller Lernprozesse hat.

Insgesamt zeigt sich der Bedarf, diese Bereiche in der Entwicklungsund Forschungsarbeit in interdisziplinären Kooperationen, an denen z.B. Lehrkräfte, Expertinnen und Experten der Fachwissenschaften, Medienpädagoginnen und -pädagogen, Mediendidaktikerinnen und -didaktiker insbesondere Machine Learning- und IT-Expertinnen und -Experten beteiligt sind, zu integrieren. Aufgrund der breit aufgestellten erforderlichen Expertise - insbesondere bezogen auf die Integration von Machine Learning-Algorithmen, den Aufbau kollaborativer Schnittstellen und die Gestaltung von Lernwelten - werden Austauschplattformen mit hochwertigen digitalen Unterrichtsmaterialien in Zukunft an Relevanz gewinnen. Dadurch wird es möglich sein, Lehrkräfte bei der komplexen Aufgabe zu unterstützen, (digitale) Unterrichtsmaterialien zu gestalten und LehrLern-Arrangements gekonnt so zu orchestrieren, dass der Einsatz digitaler Medien mit Blick auf die Lernunterstützung effektiviert werden kann.

\section{Literatur}

Adesope, Olusola O. und John C. Nesbit. 2012. «Verbal redundancy in multimedia learning environments: A meta-analysis.» Journal of Educational Psychology 104 (1): 250-63. https://doi.org/10.1037/a0026147.

Baecker, Dirk. 2017. «Wie verändert die Digitalisierung unser Denken und unseren Umgang mit der Welt?». In Handel 4.0, hrsg. von Rainer Gläß und Bernd Leukert, 3-24. Berlin, Heidelberg: Springer.

Barak, Miri und Yehudit J. Dori. 2011. «Science education in primary schools: Is an animation worth a thousand pictures?». Journal of Science Education and TechnologY 20 (5): 608-20. https://doi.org/10.1007/s10956-011-9315-2. 
Birk, Max V., Cheralyn Atkins, Jason T. Bowey und Regan L. Mandryk. 2016. «Fostering intrinsic motivation through avatar identification in digital games.» In Proceedings of the $2016 \mathrm{CHI}$ Conference on Human Factors in Computing Systems, hrsg. von Jofish Kaye, Allison Druin, Cliff Lampe, Dan Morris und Juan P. Hourcade, 2982-95. New York, NY, USA: ACM.

Black, Paul und Dylan Wiliam. 2009. «Developing the theory of formative assessment.» Educational Assessment, Evaluation and Accountability 21 (1): 5-31. https://doi.org/10.1007/s11092-008-9068-5.

Brand-Gruwel, Saskia, Iwan Wopereis und Amber Walraven. 2009. «A descriptive model of information problem solving while using internet.» Computers \& Education 53 (4): 1207-17. https://doi.org/10.1016/j.compedu.2009.06.004.

Calvo, Paco und Antoni Gomila, Hrsg. 2008. Handbook of cognitive science: An embodied approach. 1. Aufl. Amsterdam, Boston, London: Elsevier Science.

Clark, Douglas B., Emily E. Tanner-Smith und Stephen S. Killingsworth. 2016. «Digital games, design, and learning: A systematic review and meta-analysis.» Review of Educational Research 86 (1): 79-122.

Deci, Edward L. und Richard M. Ryan. 1985. Intrinsic motivation and self-determination in human behavior. Boston, MA: Springer.

Delgado, Pablo, Cristina Vargas, Rakefet Ackerman und Ladislao Salmerón. 2018. «Don't throw away your printed books: A meta-analysis on the effects of reading media on reading comprehension.» Educational Research Review 25: 2338. https://doi.org/10.1016/j.edurev.2018.09.003.

Deterding, Sebastian, Dan Dixon, Rilla Khaled und Lennart Nacke. 2011. «From game design elements to gamefulness.» In Proceedings of the 15th International Academic MindTrek Conference on Envisioning Future Media Environments - MindTrek ,11, hrsg. von Artur Lugmayr, Heljä Franssila, Christian Safran und Imed Hammouda, 9-15. New York, NY, USA: ACM Press.

Doshi-Velez, Finale und Been Kim. 2017. «Towards a rigorous science of interpretable machine learning.» Unveröffentlichtes Manuskript. http://arxiv.org/ pdf/1702.08608v2.

Eckardt, Linda und Susanne Robra-Bissantz. 2016. «Lost in Antarctica: Designing an information literacy game to support motivation and learning Success.» In Tackling Society's Grand Challenge with Design Science: 11th International Conference, DESRIST 2016, St. John's, NL, Canada, May 23-25, 2016 : Proceedings. Bd. 9661, hrsg. von Jeffrey Parsons, 202-6. Lecture notes in computer science information systems and applications, incl. Internet/Web, and HCI 9661. Cham, Heidelberg: Springer.

Efron, Bradley und Trevor Hastie. 2016. Computer Age Statistical Inference: Cambridge University Press.

Eickelmann, Birgit und Mario Vennemann. 2013. «Digitale Medien in der Grundschule Deutschland und Österreich im Spiegel der internationalen Vergleichsstudie TIMSS 2011.» Medienimpulse, 51 (2): 1-27.

Engebretsen, Martin. 2000. «Hypernews and Coherence.» Nordicom Review 21 (2): 209-25. https://doi.org/10.1515/nor-2017-0381. 
Franke-Braun, Gudrun. 2008. Aufgaben mit gestuften Lernhilfen: Ein Aufgabenformat zur Förderung der sachbezogenen Kommunikation und Lernleistung für den naturwissenschaftlichen Unterricht. Studien zum Physik- und Chemielernen 88. Berlin: Logo-Verl. Zugl. Kassel, Univ., Diss., 2008.

Gerth, Sabrina, Annegret Klassert, Thomas Dolk, Michael Fliesser, Martin H. Fischer, Guido Nottbusch und Julia Festman. 2016. "Is handwriting performance affected by the writing surface? Comparing preschoolers', second graders', and adults' writing performance on a tablet vs. paper.» Frontiers in Psychology 7:1308. https://doi.org/10.3389/fpsyg.2016.01308.

Ginns, Paul. 2005. "Meta-analysis of the modality effect.» Learning and Instruction 15 (4): 313-31. https://doi.org/10.1016/j.learninstruc.2005.07.001.

Hasler, Béatrice Susanne, Bernd Kersten und John Sweller. 2007. «Learner control, cognitive load and instructional animation.» Applied Cognitive PsychologY. 21 (6): 713-29. https://doi.org/10.1002/acp.1345.

Hattie, John. 2012. Visible learning for teachers: Maximizing impact on learning. London: Routledge.

Hattie, John und Helen Timperley. 2007. «The power of feedback.» Review of Educational Research 77 (1): 81-112. https://doi.org/10.3102/003465430298487.

Herrlinger, Simone. 2012. «Multimedia learning in primary schools.» Duisburg, Essen, Universität Duisburg-Essen, Diss., 2011, Universitätsbibliothek Duisburg-Essen.

Herrlinger, Simone, Tim N. Höffler, Maria Opfermann und Detlev Leutner. 2017. «When do pictures help learning from expository text? Multimedia and modality effects in primary schools.» Research in Science Education 47 (3): 685704. https://doi.org/10.1007/s11165-016-9525-y.

Herzig, Bardo. 2014. Wie wirksam sind digitale Medien im Unterricht? Gütersloh: Bertelsmann Stiftung. https://www.bertelsmann-stiftung.de/fileadmin/files/ BSt/Publikationen/GrauePublikationen/Studie_IB_Wirksamkeit_digitale_ Medien_im_Unterricht_2014.pdf (zuletzt aufgerufen am 30.11.2019).

Huber, Stephan Gerhard, Paula Sophie Günther, Nadine Schneider, Christoph Helm, Marius Schwander, Julia A. Schneider und Jane Pruitt. 2020. COVID-19 und aktuelle Herausforderungen in Schule und Bildung: Erste Befunde des SchulBarometers in Deutschland, Österreich und der Schweiz. Münster: Waxmann.

Kornmann, Jessica, Yvonne Kammerer, Ingo Zettler, Ulrich Trautwein und Peter Gerjets. 2016. «Hypermedia exploration stimulates multiperspective reasoning in elementary school children with high working memory capacity: A tablet computer study.» Learning and Individual Differences 51:273-83. https:// doi.org/10.1016/j.lindif.2016.08.041.

Kulik, James A. und J. D. Fletcher. 2016. «Effectiveness of intelligent tutoring systems.》 Review of Educational Research 86 (1): 42-78. https://doi. org/10.3102/0034654315581420. 
Kultusministerkonferenz [KMK], Sekretariat, Hrsg. 2016. Strategie der Kultusministerkonferenz "Bildung in der digitalen Welt". Berlin: KMK. https://www.kmk. org/fileadmin/Dateien/pdf/PresseUndAktuelles/2017/Strategie_neu_2017_ datum_1.pdf (zuletzt aufgerufen am 29.11.2019)

Lawless, Kimberley A. und P. G. Schrader. 2008. "Where do we go now? Understanding research on navigation in complex digital environments." In Handbook of New Literacies hrsg. von Julie Coiro, Michele Knobel, Colin Lankshear und Donald. J. Leu, 267-296. Hillsdale: Lawrence Erlbaum Associates.

Leahy, Wayne und John Sweller. 2011. «Cognitive load theory, modality of presentation and the transient information effect.» Applied Cognitive Psychology 25 (6): 943-51. https://doi.org/10.1002/acp.1787.

Ma, Wenting, Olusola O. Adesope, John C. Nesbit und Qing Liu. 2014. «Intelligent tutoring systems and learning outcomes: A meta-analysis.» Journal of Educational Psychology 106 (4): 901-18. https://doi.org/10.1037/a0037123.

Mangen, Anne und Don Kuiken. 2014. «Lost in an iPad: Narrative engagement on paper and tablet» Scientific Study of Literature 4 (2): 150-77. https://doi. org/10.1075/ssol.4.2.02man.

Mangen, Anne, Liss Gøril Anda, Gunn H. Oxborough und Kolbjørn Kallesten Brønnick. 2015. «Handwriting versus keyboard writing: Effect on word recall.» Journal of Writing Research 7 (2): 227-47. https://doi.org/10.17239/jowr2015.07.02.1.

Mangen, Anne, Gérard Olivier und Jean-Luc Velay. 2019. "Comparing comprehension of a long text read in print book and on kindle: Where in the text and when in the story?». Frontiers in Psychology 10: 38. https://doi.org/10.3389/ fpsyg.2019.00038.

Martschinke, Sabine. 2015. «Facetten adaptiven Unterrichts aus der Sicht der Unterrichtsforschung.» In Lernprozessbegleitung und adaptives Lernen in der Grundschule, hrsg. von Katrin Liebers, Brunhild Landwehr, Anne Marquardt und Kezia Schlotter, 15-32. Wiesbaden: Springer.

Mayer, Richard E. 2009. Multimedia Learning. Cambridge: Cambridge University Press.

Mayer, Richard E. 2014. The Cambridge Handbook of Multimedia Learning. Cambridge: Cambridge University Press.

Meurers, Detmar, Kordula de Kuthy, Florian Nuxoll, Björn Rudzewitz und Ramon Ziai. 2019. «Scaling up intervention studies to investigate real-life foreign language learning in school.» Annual Review of Applied Linguistics 39:161-88. https://doi.org/10.1017/S0267190519000126.

Molnar, Christoph. 2018. «iml: An R package for Interpretable Machine Learning.» Journal of Open Source Software 3 (26): 786. https://doi.org/10.21105/joss.00786.

Naumann, Johannes und Ladislao Salmerón. 2016. «Does navigation always predict performance? Effects of navigation on digital reading are moderated by comprehension skills.» International Review of Research in Open and Distributed Learning 17 (1). https://doi.org/10.19173/irrodl.v17il.2113. 
Nkambou, Roger, Jacqueline Bourdeau und Riichiro Mizoguchi. 2010. Advances in intelligent tutoring systems 308. Berlin, Heidelberg: Springer.

Oser, Fritz K. und Franz J. Baeriswyl. 2001. «Choreographies of teaching: Bridging instruction to learning.» In Handbook of research on teaching, hrsg. von Virginia Richardson. 4. Aufl., 1031-65. Washington: American Educational Research Association.

Renkl, Alexander und Robert K. Atkinson. 2007. "Interactive learning environments: contemporary issues and trends. An introduction to the special issue.» Educational Psychology Review 19 (3): 235-38. https://doi.org/10.1007/s10648007-9052-5.

Rouet, Jean-François, Christine Ros, Antonine Goumi, Mônica Macedo-Rouet und Jérôme Dinet. 2011. "The influence of surface and deep cues on primary and secondary school students' assessment of relevance in web menus.» Learning and Instruction 21 (2): 205-19. https://doi.org/10.1016/j.learninstruc.2010.02.007.

Ryan, Richard M., C. Scott Rigby und Andrew Przybylski. 2006. «The motivational pull of video games: A self-determination theory approach.» Motivation and Emotion 30 (4): 344-60. https://doi.org/10.1007/s11031-006-9051-8.

Salmerón, Ladislao und Victoria García. 2011. «Reading skills and children's navigation strategies in hypertext.» Computers in Human Behavior 27 (3): 1143-51. https://doi.org/10.1016/j.chb.2010.12.008.

Salmerón, Ladislao, Walter Kintsch und José J. Cañas. 2006. «Reading strategies and prior knowledge in learning from hypertext.» Memory and Cognition 34 (5): 1157-71. https://doi.org/10.3758/BF03193262.

Salmerón, Ladislao, Helge I. Strømsø, Yvonne Kammerer, Marc Stadtler und Paul van den Broek. 2018. «Comprehension processes in digital reading.» In Learning to Read in a Digital World. Bd. 17, hrsg. von Mirit Barzillai, Jenny Thomson, Sascha Schroeder und Paul van den Broek, 91-120. Amsterdam: John Benjamins Publishing Company.

Schaumburg, Heike. 2015. Chancen und Risiken digitaler Medien in der Schule. Medienpädagogische und -didaktische Perspektiven. Gütersloh: Bertelsmann Stiftung. https://www.bertelsmann-stiftung.de/fileadmin/files/BSt/Publikationen/GrauePublikationen/Studie_IB_Chancen_Risiken_digitale_Medien_2015.pdf (zuletzt aufgerufen am 30.11.2019).

Schmidt, Christin und Katrin Liebers. 2015. "Formatives Assessment an Grundschulen - Praxis und Bedingungsfaktoren.» In Lernprozessbegleitung und adaptives Lernen in der Grundschule, hrsg. von Katrin Liebers, Brunhild Landwehr, Anne Marquardt und Kezia Schlotter, 133-38. Wiesbaden: Springer.

Schüler, Anne, Katharina Scheiter und Florian Schmidt-Weigand. 2011. «Boundary conditions and constraints of the modality effect.» Zeitschrift für Pädagogische Psychologie 25 (4): 211-20. https://doi.org/10.1024/1010-0652/a000046.

Schütze, Birgit, Elmar Souvignier und Marcus Hasselhorn. 2018. «Stichwort Formatives Assessment.» Zeitschrift für Erziehungswissenschaft 21 (4): 697715. https://doi.org/10.1007/s11618-018-0838-7. 
Sidi, Yael, Yael Ophir und Rakefet Ackerman. 2016. "Generalizing screen inferiority - does the medium, screen versus paper, affect performance even with brief tasks?». Metacognition Learning 11 (1): 15-33. https://doi.org/10.1007/ s11409-015-9150-6.

Souvignier, Elmar und Marcus Hasselhorn. 2018. "Formatives Assessment.» Zeitschrift für Erziehungswissenschaft 21 (4): 693-96. https://doi.org/10.1007/ s11618-018-0839-6.

Spires, Hiller A., Jonathan P. Rowe, Bradford W. Mott und James C. Lester. 2011. "Problem solving and game-based learning: Effects of middle grade students' hypothesis testing strategies on learning outcomes.» Journal of Educational Computing Research 44 (4): 453-72. https://doi.org/10.2190/EC.44.4.e.

Spiro, Rand, Paul J. Feltovich, Michael J. Jacobson und Richard Lorne Coulson. 1992. "Cognitive flexibility, constructivism, and hypertext: Random access instruction for advanced knowledge acquisition in ill-structured domains." Educational Technology 31 (5): 24-33.

Squire, Kurt, James Paul Gee und Henry Jenkins. 2011. Video games and learning: Teaching and participatory culture in the digital age. Technology, educationconnections the TEC series. New York, NY: Teachers College Press.

Steenbergen-Hu, Saiying und Harris Cooper. 2014. "A meta-analysis of the effectiveness of intelligent tutoring systems on college students' academic learning.» Journal of Educational Psychology 106 (2): 331-47. https://doi.org/10.1037/ a0034752.

Tobar-Muñoz, Hendrys, Silvia Baldiris und Ramon Fabregat. 2017. «Augmented Reality game-based learning: enriching students' experience during reading comprehension activities.» Journal of Educational Computing Research 55 (7): 901-36. https://doi.org/10.1177/0735633116689789.

Totter, Alexandra, Julia Häbig, Daniela Müller-Kuhn, und Enikö Zala-Mezö. 2020. «Zwischen traditionellem Schulbuch und hybridem Lehrmittel. Bedingungen, Möglichkeiten und Herausforderungen von Lernen und Lehren in einer digitalen Welt». Zeitschrift MedienPädagogik 17 (Jahrbuch Medienpädagogik), 169-193. https://doi.org/10.21240/mpaed/jb17/2020.04.30.X.

Vogel, Jennifer J., David S. Vogel, Jan Cannon-Bowers, Clint A. Bowers, Kathryn Muse und Michelle Wright. 2006. "Computer gaming and interactive simulations for learning: A meta-analysis.» Journal of Educational Computing Research 34 (3): 229-43. https://doi.org/10.2190/FLHV-K4WA-WPVQ-HoYM.

Vögelin, Cristina, Thorben Jansen, Stefan D. Keller und Jens Möller. 2018. «The impact of vocabulary and spelling on judgments of ESL essays: an analysis of teacher comments.» The Language Learning Journal 49 (2): 1-17. https://doi.org /10.1080/09571736.2018.1522662.

Voss, Andreas. 2006. Print- und Hypertextlesekompetenz im Vergleich: Eine Untersuchung von Leistungsdaten aus der internationalen Grundschul-Lese-Untersuchung (IGLU) und der Ergänzungsstudie Lesen am Computer (LaC). Empirische Erziehungswissenschaft 1. Münster: Waxmann. Zugl. Hamburg, Univ., Diss., 2004 u.d.T. Voss, Andreas: Ein Vergleich von Print- und Hypertextlesekompetenzen anhand der Studien IGLU und LaC. 
Wang, Margaret C., Patricia Gennari und Hersholt C. Waxman. 1985. The adaptive learning environments model: Design, implementation, and effects. US Department of Education, National Institute of Education Educational Resources Information Center (ERIC).

Wedel-Wolff, Annegret von. 2011. Üben im Rechtschreibunterricht der Grundschule: Systematische Vorschläge für die Klassen 2 - 4. 1. Aufl. [Nachdr.]. Praxis Pädagogik. Braunschweig: Westermann.

Wigfield, Allan und Jacquelynne S. Eccles. 1992. "The development of achievement task values: A theoretical analysis.» Developmental Review 12 (3): 265310. https://doi.org/10.1016/0273-2297(92)90011-P.

Wong, Anna, Wayne Leahy, Nadine Marcus und John Sweller. 2012. «Cognitive load theory, the transient information effect and e-learning.» Learning and Instruction 22 (6): 449-57. https://doi.org/10.1016/j.learninstruc.2012.05.004.

Wouters, Pieter, Christof van Nimwegen, Herre van Oostendorp und Erik D. van der Spek. 2013. «A meta-analysis of the cognitive and motivational effects of serious games.» Journal of Educational Psychology 105 (2): 249-65. https://doi. org/10.1037/a0031311.

Zehner, Fabian, Christine Sälzer und Frank Goldhammer. 2016. "Automatic coding of short text responses via clustering in educational assessment.» Educational and Psychological Measurement 76 (2): 280-303. https://doi. org/10.1177/0013164415590022.

Zorn, Isabel. 2011. «Zur Notwendigkeit der Bestimmung einer auf Digitale Medien fokussierten Medienkompetenz und Medienbildung.» MedienPädagogik 20: 175-209. https://doi.org/10.21240/mpaed/20/2011.09.19.X. 